\title{
Design and impact of a commercial educational robotic exoskeleton
}

\author{
Volker Bartenbach and Camila Shirota
}

\begin{abstract}
Robotics has been shown to effectively engage students in STEM education. Wearable robots have the potential to appeal to a wider audience due to their inclusion of biological disciplines; however, no educational tool is currently available. Here, we present the design and impact of the EduExo, an educational robotic exoskeleton kit. As requirements, the kit should stand alone, require minimal knowledge and additional equipment, and appeal to a wide audience. User feedback and requests within the first year of availability suggest that there is interest for such kits, within and outside of formal educational settings. We present a few examples of use cases. Further, potential financial barriers seem to be outweighed by the convenience of a ready-to-use kit. We believe the framework and experiences presented here are valuable for future developers of educational robotics kits.
\end{abstract}

\section{INTRODUCTION}

$\mathrm{S}$ CIENCE, Technology, Engineering and Math (STEM) education is an increasing priority in multiple countries, as it develops abilities beyond technical disciplines that are critical for societal life [1]. Although interest and engagement in these disciplines are notoriously challenging, project-based robotics has shown potential as an effective educational tool, especially for younger students [1].

An emerging subfield of robotics that could increase interest in STEM are wearables. Robotic exoskeletons, in particular, have seen increasing application in healthcare and industry. Their interface with a biological system increases the appeal to less technically-inclined students, and the impact of applications beyond the classroom could boost interest and engagement. However, in contrast to general robotics, there are few if any educational tools based on wearable robots, hindering their integration into educational programs due to the lack of readily-available material [2].

The goal of this paper is to present the development framework of a robotic exoskeleton kit for educational purposes, targeted at a general audience. We further present its impact one year in, and experiences gained, which could be used to inform future educational material.

\section{MATERIALS AND METHODS}

The goal was to develop and offer a kit that develops an

The EduExo is a commercial product developed and sold by Beyond Robotics $\mathrm{GmbH}$, Switzerland. The development was supported by private funds from the EduExo team, 175 people through a Kickstarter Campaign, and the Wearable Robotics Association Innovation Challenge 2017.

V. Bartenbach is with Beyond Robotics GmbH, Switzerland (info@beyond-robotics.com).

C. Shirota is with the Rehabilitation Engineering Lab, ETH Zürich, Switzerland (camila.shirota@hest.ethz.ch), and Advisor in science and technology for EduExo. introductory understanding about exoskeleton technology and its applications, together with experience building and programming a functioning robotic exoskeleton. To reach the widest audience, financial costs were critical and kept to a minimum. Further, the material should stand alone and allow unsupervised learning, i.e., without an instructor. The result is the EduExo - a robotic exoskeleton kit that users assemble and program themselves (Fig. 1), taking a projectbased approach to learning about the design and construction of exoskeletons. It is composed of 3 parts (described below): the exoskeleton, the handbook, and the website.

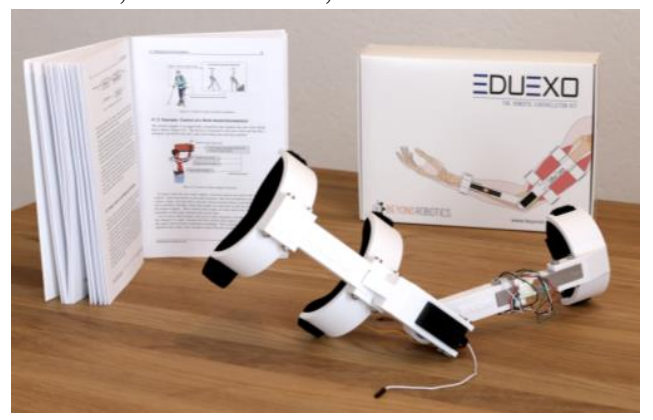

Fig. 1. The EduExo kit: it contains the handbook and all parts to assemble and program the exoskeleton.

\section{A. The Exoskeleton}

To keep concepts simple and decrease hardware malfunction, a one degree-of-freedom elbow exoskeleton design was chosen (Fig. 2). The hardware combines off-theshelf components (motor, force sensor and microcontroller) with a 3D-printed exoskeleton structure and cuff interfaces.

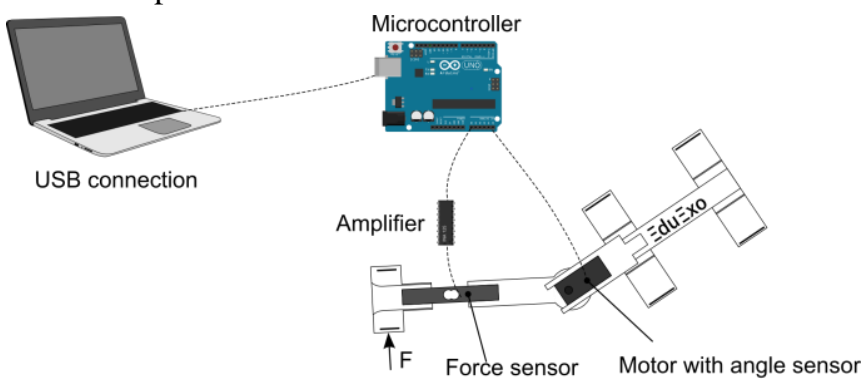

Fig. 2. Overview of the main EduExo hardware components. The PC is not included in the kit.

To appeal to different technical skillsets and budgets, two different versions of the kit were created. The Boxed version contains the printed handbook and all mechanical and electronic components. In contrast, the Maker Edition is entirely digital, and requires users to 3D-print the exoskeleton structure themselves and order all parts, thus including manufacturing and part supply into the exoskeleton project. An add-on kit for myoelectric control 
was also developed, to introduce motor control concepts.

To increase accessibility of the kit, additional equipment and resources needed to assemble and use the hardware were kept to a minimum. Only a computer, a soldering iron, a USB A/B cable, hex keys and a flat-blade screwdriver are required - most of which are readily available at homes, or can be easily acquired. Additionally, users have to install the Arduino IDE and the Unity 3D game engine, which are available for free for non-commercial use.

\section{B. The Handbook}

The handbook covers the main aspects of exoskeleton technology, introducing users to many disciplines involved in robotic exoskeleton design. It consists of 5 chapters; the first is a general introduction to the field, with applications. The remaining chapters introduce one aspect of exoskeleton technology each (Anatomy and Mechanics; Electronics and Software; Control Systems; and VR and Games), and with theoretical background followed by a set of tutorials to progressively assemble the hardware throughout the book.

To present the information in an appealing and accessible way even to people without previous specialized knowledge, the handbook contains around 40 illustrations, 20 photos, 10 screenshots, 20 code listings and 20 info boxes. A slide deck is also supplied in Classroom Sets, to support instructors.

\section{The Website}

A website (www.eduexo.com) complementary to the handbook was created, to provide multimedia content and articles about exoskeletons. The website also functions as a channel to showcase users' EduExo-related projects, and a platform to exchange ideas and build a community. This was thought to be of special importance for users that do not have a chance to discuss their projects, e.g., in a classroom.

\section{Financial model}

To ensure continuation of the project, a for-profit organization model was chosen. By being profitable, the project is independent of public and third-party funding frameworks and timelines, and can sustain its own continued development. Initial funding for the project was partially acquired through a pre-ordering crowdfunding campaign on Kickstarter. This also allowed evaluation of demand and interest before completely finalizing the kit.

\section{RESULTS}

As of September 2018 (one year after release), several hundred EduExos have been shipped to more than 40 countries. The largest number of devices are in North America, Europe and Asia (especially South Korea).

Four main use cases of the kit could be indirectly identified based on inquiries and feedback: private use, in extracurricular activities, as a technology demonstrator, and as part of a curriculum. Private users are hobbyists, makers and students who build the EduExo at home, by themselves or with friends. The EduExo has also been used in extracurricular activities, such as exoskeleton workshops given by robotics clubs [3], and to present exoskeleton technology to wider audiences. For example, it is used in the Cybathlon Experience program that aims to present novel rehabilitation and assistive technologies to the general public [4].

The second main use is as part of formal courses with rewarded credits, from high school to undergraduate level. For example, a vocational school in Switzerland is using the device to familiarize future healthcare professionals with new technologies they will encounter in their profession. The EduExo has also been used as a base platform to which engineering students added their own customizations as a semester project [5]. Finally, it was used as a measurement device for advanced biomedical engineering concepts [6].

One challenge reported thus far is the integration of the material into traditional high school curricula. Further, language is a barrier for users in non-English speaking countries, especially for younger students (up to high school) and students in professions that operate mainly in local languages (e.g., nurses, therapists).

\section{DISCUSSION AND CONCLUSION}

Robotic exoskeletons are increasingly relevant and have the potential to attract a wide audience to STEM education. We presented the design and first year of experience with an educational kit, which could inform future endeavours.

So far, the acceptance of the EduExo as a tool to learn about and teach exoskeleton technology has been promising, showing that there is worldwide interest for such devices. Further, its use in diverse formal education settings suggests the need for easily-accessible educational tools to train students at various levels and with different backgrounds.

Regarding the decision for a commercial rather than an open source project, we have a positive conclusion. It has ensured availability of the kit and the continuation of the project independent of academic funding cycles. Also, for many educators, the time savings associated with a prepackaged kit seems to be more important than the financial barrier, as the Boxed version is far more popular with academic users than the more involved Maker Edition.

Finally, language was a significant barrier for many target user groups, and should be considered when developing such devices. This, and the availability over the internet, could have limited the reach and use of this device, and should be considered when interpreting our findings.

\section{REFERENCES}

[1] S. Marginson, R. Tytler, B. Freeman, and K. Roberts, STEM: Country comparisons, 2013.

[2] M. Saleiro, B. Carmo, J. M. F. Rodrigues, and J. M. H. du Buf, "A Low-Cost Classroom-Oriented Educational Robotics System," vol. 8239, pp. 74-83, 2013.

[3] http://www.tetraryerson.ca/workshops/

[4] http://www.cybathlon.ethz.ch/road-to-2020/cybathlon-experience.html

[5] https://www.eduexo.com/community-projects/elex-exoskeleton/

[6] A. M. Gonabadi and P. Malcolm, "Tutorial: Using EduExo to visualize electromechanical delay in $m$. biceps brachii," University of Nebraska Omaha2018. 